\title{
Técnica inovadora para tratamento de dente permanente imaturo - Revascularização
}

\section{pulpar}

\author{
Innovative techniques for immature permanent teeth treatment - Pulp revascularization
}

Técnicas innovadoras para el tratamiento de dientes permanentes inmaduros - Revascularización

Nathália Alves da Silva

ORCID: https://orcid.org/0000-0002-2362-3679 Universidade Federal de Alagoas, Brasil E-mail: nathaliaalves1202@gmail.com

Andréa Albuquerque Lima

ORCID: https://orcid.org/0000-0001-5861-3799 Universidade Federal de Uberlândia, Brasil

E-mail: dra.andrealima@gmail.com

Phillip Lucas Ricardo Pereira ORCID: https://orcid.org/0000-0003-0911-4672 Faculdade Herrero, Brasil

E-mail: phillippereira@gmail.com

Clóvis Stephanno Pereira Bueno ORCID: https://orcid.org/0000-0001-9149-1674 Faculdade Herrero, Brasil

E-mail: clovisspbueno@gmail.com

Daniel Pinto de Oliveira

ORCID: https://orcid.org/0000-0003-1992-9920 Universidade Federal de Alagoas, Brasil E-mail: dpoendo@yahoo.com.br

Leopoldo Cosme Silva

ORCID: https://orcid.org/0000-0002-5755-1933 Universidade Federal de Alagoas, Brasil

E-mail: leopoldocosme@gmail.com

Laís Christina Pontes Espíndola

ORCID: https://orcid.org/0000-0002-1023-7891 Universidade Federal de Alagoas, Brasil E-mail:laisespindola@hotmail.com

Wedja Karla Florêncio Neves

ORCID: https://orcid.org/0000-0002-3978-4274

Faculdade de Odontologia de Caruaru, Brasil E-mail:wedjaneves@hotmail.com

Dyana dos Santos Fagundes

ORCID: https://orcid.org/0000-0002-7427-4114

Universidade Federal de Alagoas, Brasil

E-mail: dydyana_fagundes@hotmail.com

\section{Resumo}

O tratamento de dentes com rizogênese incompleta e necrose pulpar representa um grande desafio para a terapia endodôntica, principalmente pela fragilidade das paredes radiculares desses dentes. Uma terapêutica que pode ser realizada é a revascularização pulpar com o objetivo de substituir estruturas danificadas, incluindo estruturas dentinárias e radiculares, bem como células do complexo dentino-pulpar com um tecido que permita a continuação da formação da raiz em espessura e tamanho. Esses procedimentos demonstraram ser capazes de promover o desenvolvimento continuado da raiz, aumentando assim a espessura e a resistência das raízes anteriormente finas e propensas à fratura. Este trabalho tem como objetivo realizar, através de uma revisão da literatura, um estudo sobre a revascularização pulpar e sua importância clínica no tratamento de dentes acometidos por necrose pulpar e cuja formação apical ainda não se completou. Para este estudo, foi realizado um levantamento na plataforma PubMed, em que foram incluídos artigos publicados entre 2004 e 2021. A revascularização pulpar pode ser considerada como uma alternativa promissora como tratamento para dentes com rizogênese incompleta e necrose pulpar, tendo vantagens potenciais em relação ao tradicional processo de apicificação.

Palavras-chave: Endodontia; Endodontia regenerativa; Necrose pulpar. 


\begin{abstract}
The treatment of incomplete rizogenesis and pulp necrosis presents a great challenge for endodontic therapy, mainly due to the fragility of the root walls of these teeth. The main therapy chosen is pulp revascularization with the aim of replacing damaged structures, including dentin and root structures, as well as cells of the dentin-pulp complex with a tissue that allows the root formation to continue in thickness and size. These procedures have been shown to be capable of promoting continuous root development, thus increasing the thickness and strength of previously thin fracture-prone roots. The objective of this study is to carry out a review of the literature on pulpal revascularization and the clinical importance in the treatment of teeth affected by pulpal necrosis and whose apical formation has not yet been completed. A survey was performed on the PubMed platform and included articles published between 2004 and 2020. Pulp revascularization may be considered a promising alternative as the treatment for teeth with incomplete rizogenesis and pulp necrosis, the blinds struggle with the traditional apicification process.
\end{abstract}

Keywords: Endodontics; Regenerative endodontics; Pulp necrosis.

\title{
Resumen
}

El tratamiento de dientes con rizogénesis incompleta y necrosis pulpar representa un gran desafío para la terapia endodóntica, principalmente debido a la fragilidad de las paredes radiculares de estos dientes. Una terapia que se puede realizar es la revascularización pulpar para reemplazar las estructuras dañadas, incluidas la dentina y las estructuras radiculares, así como las células del complejo dentino-pulpar, con tejido que permita la continuación de la formación radicular en grosor y tamaño. Se ha demostrado que estos procedimientos pueden promover el desarrollo continuo de las raíces, aumentando así el grosor y la resistencia de las raíces que antes eran delgadas y propensas a fracturas. Este trabajo tiene como objetivo realizar, a través de una revisión de la literatura, un estudio sobre la revascularización pulpar y su importancia clínica en el tratamiento de dientes afectados por necrosis pulpar y cuya formación apical aún no se ha completado. Para este estudio se realizó una encuesta en la plataforma PubMed, que incluyó artículos publicados entre 2004 y 2021. La revascularización pulpar puede considerarse una alternativa prometedora como tratamiento para dientes con conductos radiculares incompletos y necrosis pulpar, con posibles ventajas sobre el proceso tradicional de apicificación.

Palabras clave: Endodoncia; Endodoncia regenerativa; Necrosis pulpar.

\section{Introdução}

O dente permanente humano tem seu desenvolvimento radicular normalmente completado de 1 a 4 anos após a erupção na boca e nesta fase é considerado imaturo. Em casos de ocorrência de dano irreversível à polpa dentária devido à cárie ou trauma, o desenvolvimento radicular é comprometido, resultando em um dente com ápice incompletamente formado, paredes dentinárias finas e relação coroa-raiz inapropriada (Chen et al., 2015).

Em dentes imaturos necrosados existem dois tipos de tratamento, a apicificação e a revascularização pulpar. A primeira proposta, realizada através da inserção de um plug de MTA no ápice radicular, pode trazer 74\% a $100 \%$ de sucesso (Jo et al., 2002; Al Ansary et al., 2009; Botero et al., 2017). No entanto, não possibilita a regeneração fisiológica do complexo dentinopulpar, e assim, não permite a completa formação radicular (Jeeruphan et al., 2012; Eramo et al., 2018).

A técnica de revascularização pulpar ou procedimentos endodônticos regenerativos, por sua vez, tem o objetivo de preencher o canal radicular com um tecido que permita a continuação da formação da raiz em espessura e tamanho. Suas vantagens recaem na possibilidade de completo desenvolvimento radicular, reforçando as paredes dentinárias pela deposição de tecido duro, fortalecendo assim a raiz contra fraturas (Banchs \& Trope, 2004) . Pode-se falar que são procedimentos biológicos planejados para substituir os tecidos danificados, incluindo as estruturas dentinárias e radiculares, bem como as células do complexo polpa-dentina (Montero-Miralles et al., 2018).

Como uma abordagem alternativa, a Endodontia Regenerativa visa regenerar tecidos semelhantes à polpa dental usando duas estratégias possíveis: transplante celular e cell homing. O primeiro requer células-tronco exogenamente transplantadas, sendo um procedimento complexo e de alto custo. O segundo emprega as células endógenas do hospedeiro para conseguir a reparação/regeneração tecidual, tornando-se, clinicamente, uma alternativa mais viável (Eramo et al., 2018).

A Associação Americana de Endodontistas (AAE) lançou um protocolo para esta técnica baseado na seguinte sequência: descontaminação do canal radicular com mínimo de desgaste das paredes; medicação intracanal por 21 dias 
estimando retorno sem sinais ou sintomas; remoção da medicação em segunda sessão; irrigação com 20 mL de EDTA 17\%; indução de sangramento via periápice através de transpasse de lima manual $2 \mathrm{~mm}$ além do comprimento do dente, ou inserção de PRF, ou matriz de fibrina autóloga; proteção do tecido através de selamento dos 3 a $4 \mathrm{~mm}$ cervicais da raiz com material biocompatível e alta capacidade seladora, evitando microinfiltraçao bacteriana (American Association of Endodontists, 2016).

Para atingir o sucesso deste tratamento é primordial a ausência de microrganismos no interior do canal, enaltecendo a importância de total desinfecção para o sucesso dos casos de regeneração pulpar (Verma et al., 2016), bem como o impedimento de novas contaminações bacterianas através de adequado selamento do terço cervical. Os antibióticos têm sido amplamente utilizados em casos de procedimentos endodônticos regenerativos (PERS) nos canais radiculares (Montero-Miralles et al., 2018).

A AAE propõe como material selador em casos de revascularização pulpar, o MTA Branco e o Biodentine, materiais à base de silicato de cálcio com boa capacidade seladora e boas propriedades físico-químicas, trazendo as vantagens de serem carreadores de íons cálcio, o que estimula a produção de tecido duro (American Association of Endodontists, 2016).

A revascularização pode ser uma técnica a ser preconizada para o tratamento de dentes imaturos, sendo o presente estudo uma revisão de literatura importante para a consolidação de informações, permitindo aos endodontistas a compreensão dos objetivos, materiais em uso e a ideia do protocolo.

\section{Metodologia}

Para a realização deste estudo foi feita uma revisão bibliográfica narrativa, em que foi realizado um levantamento bibliográfico na plataforma PubMed/MEDLINE, nos peródicos Journal of Endodontics, International Endodontic Journal, Journal of Dental Research. No qual os seguintes unitermos foram correlacionados: Endodontic, Pulp revascularization, Immature tooth, Pulp regeneration. Após análise dos critérios de inclusão (acesso ao artigo completo via internet e possuir relação direta com o objetivo do trabalho), foram selecionados artigos publicados entre 2004 e 2020. Dentre os artigos encontrados, foram incluídos aqueles que, através da análise do resumo, abordassem informações que respondessem ao objetivo inicial da pesquisa, enfatizando a importância da técnica de revascularização pulpar em dentes imaturos. Também foram incluídos artigos que foram julgados relevantes para a compreensão da técnica de revascularização pulpar. Somado aos artigos específicos, alguns autores fornecem suporte metodológicos: Estrela, C. (2018). Metodologia Científica: Ciência, Ensino, Pesquisa. Editora Artes Médicas. Pereira A. S. et al. (2018). Metodologia da pesquisa científica. [free e-book]. Santa Maria/RS. Ed. UAB/NTE/UFSM. Koche, J. C. (2011). Fundamentos de metodologia científica. Petrópolis: Vozes. Disponível. Ludke, M. \& Andre, M. E. D. A. (2013). Pesquisas em educação: uma abordagem qualitativa. São Paulo: E.P.U. Yin, R.K. (2015). O estudo de caso. Porto Alegre: Bookman.

\section{Revisão de Literatura}

Banchs e Trope (2004) foram os primeiros pesquisadores a publicar sobre a revascularização pulpar, descrevendo como uma nova técnica apresentada para revascularizar dentes permanentes imaturos com periodontite apical. Relatavam que o canal é desinfetado com irrigação abundante e uma combinação de três antibióticos. Após a conclusão do protocolo de desinfecção, o ápice é irritado mecanicamente para iniciar o sangramento no canal e produzir um coágulo de sangue ao nível da junção cemento-esmalte. Em seguida, um duplo selamento do acesso coronário é feito. Neste caso, a combinação de um canal desinfetado, uma matriz em que o novo tecido pode crescer, e um selamento coronal efetivo parece ter produzido o ambiente necessário para uma revascularização bem-sucedida.

Quatro anos depois, uma série de casos relatada por Jung et al., (2008) mostrou os resultados de 8 pacientes (idades 9-14 anos) que apresentaram 9 dentes imaturos permanentes com necrose pulpar e periodontite apical e foram tratados através da técnica de revascularização pulpar. Durante o tratamento, cinco dos dentes foram encontrados com, pelo menos, algum tecido 
pulpar vital remanescente nos sistemas de canais radiculares. Os dentes foram desinfetados com irrigação com hipoclorito de sódio $(\mathrm{NaOCl})$ e medicação com ciprofloxacino, metronidazol e minociclina, e em uma outra sessão, estes dentes foram selados com MTA e restaurados. O outro grupo de 4 dentes não evidenciou qualquer tecido de polpa vital residual. Este segundo grupo de dentes foi tratado com irrigação com $\mathrm{NaOCl}$ e medicado com ciprofloxacino, metronidazol e minociclina, seguido por um procedimento de revascularização (induzindo hemorragia para formar um coágulo sanguíneo intracanal). Os resultados mostraram que, em ambos os grupos dos pacientes, houve evidência de resultados pós-operatórios (1-5 anos). Os pacientes se mantiveram assintomáticos. A periodontite foi resolvida e houve evidência radiográfica de continuação da espessura das paredes dentinárias, fechamento ou aumento do comprimento da raiz. Concluindo, então que casos positivos fornecem um impulso para o desenvolvimento de ensaios clínicos randomizados que avaliem estes métodos.

No mesmo ano, Shah et al. (2008) avaliaram a técnica de revascularização pulpar e sua eficácia em 14 casos de infecção em dentes imaturos. O tratamento endodôntico foi iniciado e após o controle da infecção, foi realizada a revascularização. A cavidade de acesso foi selada com CIV. Os casos foram acompanhados em intervalos regulares de 3 meses com média de acompanhamento de 0,5- 3,5 anos. Como resultado, os pesquisadores observaram que a resolução radiográfica de radiolucência perirradicular foi considerada boa/excelente em 93\% (13 de 14) dos casos. Na maioria dos casos, um estreitamento apical ficou evidente. Em 2 casos, houve o espessamento de paredes dentais apicais e aumento do comprimento da raiz. A notável descoberta foi a resolução completa dos sinais e sintomas clínicos e a cicatrização apreciável de lesões periapicais em 78\% (11 de 14) dos casos. O espessamento das paredes dentinárias ficou evidente em 57\% (8/14) dos casos, e o aumento do comprimento da raiz foi observado em $71 \%$ (10/14) dos casos. Nenhum dos casos apresentou dor, reinfecção ou aumento radiográfico de alguma patologia apical preexistente. Com isso, concluiu-se que houve resultado favorável nos procedimentos de revascularização realizados neste estudo clínico.

Ding et al. (2009) examinaram o efeito de um procedimento de revascularização pulpar em dentes necróticos imaturos com periodontite apical, através da avaliação de doze pacientes, cada um com dente permanente com periodontite apical crônica ou aguda. Os dentes foram tratados em duas sessões, utilizando uma mistura triantibiótica (ciprofloxacino, metronidazol e minociclina) com o intuito de desinfectar a polpa durante uma semana. Em uma segunda sessão, foi realiza indução de coágulo de sangue no interior do canal, sobre o qual o MTA cinza foi inserido. Os pacientes foram assistidos periodicamente. Seis pacientes foram removidos do estudo (como resultado da indução de sangramento após a desinfecção do canal) e, ao invés disso, receberam um procedimento padrão de apicificação. Outros três pacientes não compareceram a nenhuma consulta. Os restantes $(n=3)$ apresentaram desenvolvimento completo da raiz, como resposta. Com base nos resultados obtidos, constatou-se que a revascularização pode ser eficaz para o tratamento de dentes permanentes imaturos com periodontite apical, quando o caso é selecionado de forma apropriada.

Shin et al., (2009) relataram um caso clínico no qual um segundo pré-molar inferior direito com polpa necrótica com presença de fístula, radioluscência perirradicular e um ápice imaturo submetido a revascularização através de uma única abordagem de tratamento. Os procedimentos de revascularização têm o potencial de reversibilidade de uma polpa parcialmente necrótica, o que pode ser benéfico para o desenvolvimento contínuo das raízes dos dentes imaturos. No entanto, não está claro quais protocolos de revascularização são os mais eficazes. Esse relato de caso realizado pelos autores detalhou o resultado de um procedimento de revascularização bem sucedido no dente 45, em um paciente de 12 anos de idade, eliminando a patologia periapical associada dentro de 19 meses. O dente foi desinfectado utilizando irrigação com $\mathrm{NaOCl}$ a $6 \%$ e Digluconato de Clorexidina a $2 \%$ em instrumentação em uma única visita. O resultado bem-sucedido deste relato de caso sugeriu que esta abordagem conservadora de tratamento de revascularização pode preservar a vitalidade das células-tronco da polpa dentária e criar um ambiente adequado para a regeneração da polpa, resultando na conclusão da maturação da raiz. A irrigação intracanal $\mathrm{NaOCl}$ a $6 \%$ e Digluconato de Clorexidina a $2 \%$ pode ajudar a preservar as células-tronco vitais restantes que são consideradas 
críticas para a revascularização da polpa e realizar um protocolo de revascularização de polpa de visita única pode ser uma opção de tratamento favorável para um dente permanente imaturo com polpa parcialmente necrótica foram pontos importantes neste caso.

Uma série de casos foi relatada por Cehreli et al. (2011), objetivando demonstrar o resultado do tratamento de revascularização com medicação intracanal de hidróxido de cálcio $[\mathrm{Ca}(\mathrm{OH}) 2]$ em molares imaturos com necrose. Primeiros molares permanentes imaturos necróticos $(\mathrm{n}=6)$ de pacientes 8-11 anos foram tratadas por um protocolo de revascularização que preconizava irrigação com $\mathrm{NaOCl}$ a 2,5\%, medicação com $\mathrm{Ca}(\mathrm{OH}) 2$ colocada no terço coronal dos canais radiculares, indução de sangramento apical (formação de coágulo) e selamento coronal com MTA branco. Dentre os dentes, 4 molares haviam sido previamente submetidos a instrumentação do canal radicular pelos dentistas referentes. O programa Image-J e TurboReg foram utilizados para a normatização das radiografias e para determinar o aumento no comprimento e largura das raízes. Os resultados mostraram que, após um período de 10 meses, todos os dentes demonstraram evidência radiográfica de cicatrização das complicações periapicais, espessamento progressivo das paredes dentinárias e desenvolvimento apical contínuo com ausência de sintomas. Dois molares não instrumentados, mostraram resposta ao teste ao frio, após 9 meses. Concluiu-se, então, que em base de um período de acompanhamento de 10 meses, os casos demonstraram um resultado favorável da revascularização em molares necróticos imaturos utilizando medicação de $\mathrm{Ca}(\mathrm{OH}) 2$ no terço coronal dos canais radiculares.

Jadhav et al. (2012) realizaram um estudo piloto clínico com o objetivo de avaliar e comparar a maturogênese induzida pela revascularização com e sem Plasma Rico em Plaquetas. Como metodologia, 20 pacientes com dentes anteriores imaturos não vitais foram categorizados aleatoriamente em 2 grupos. Após o preparo químico-mecânico, a revascularização foi induzida sem e com PRP (uma esponja de colágeno), nos grupos 1 e 2, respectivamente. Os casos foram acompanhados clinicamente e radiograficamente em intervalos de 6 e 12 meses. Os resultados mostraram que, clinicamente, todos os casos foram assintomáticos com resolução completa de sinais e sintomas. Radiograficamente, havia uma diferença marcante na cicatrização periapical, fechamento apical e espessamento da parede dentinária no grupo 2 em comparação ao grupo 1. No entanto, o alongamento da raiz foi comparável para ambos os procedimentos. Pôde-se concluir que a revascularização é um método conservador e eficaz para a indução da maturogênese em dentes extraídos e imaturos. Suplementação com PRP pode, potencialmente, melhorar o resultado biológico desejado dessa técnica de processo regenerativo.

Wigler et al. (2013) revisaram artigos com procedimentos regenerativos e de revascularização pulpar para coletar recomendações sobre as indicações, medicamentos de escolha e métodos de tratamento atualmente utilizados. Observou-se que a desinfecção da raiz e estimulação de células-tronco residuais pode induzir a neoformação de tecido duro na parede dentinária existente, dando continuidade ao desenvolvimento radicular. Com isso, concluíram que o resultado dos procedimentos de revascularização permanece um tanto imprevisível e o manejo desses dentes é um desafio, devendo então haver uma melhora nos protocolos de tratamento.

Soares et al. (2013) relataram um caso clínico de revascularização pulpar utilizando o hidróxido de cálcio e a clorexidina gel $2 \%$ para descontaminação, com objetivo de descrever uma nova proposta para revascularização do canal radicular. No relato de caso, uma menina de 9 anos, sofreu uma intrusão associada com exposição pulpar causada por uma fratura de esmalte e dentina em seu incisivo central esquerdo superior. Após o diagnóstico, o tratamento estabelecido foi a terapia de revascularização com instrumentação manual suave da região cervical e terços médios da raiz, além de medicação com hidróxido de cálcio e gel de clorexidina à $2 \%$ durante 21 dias. Na segunda sessão, um coágulo foi estimulado até o terço cervical da raiz. O Agregado Trióxido Mineral (MTA, Angelus, Brasil) foi utilizado para selamento cervical do canal. O selamento coronário foi realizado com material obturador e resina composta. Como resultado, foi observado que o espaço do canal radicular mostrou uma diminuição progressiva da largura, deposição de tecido nas paredes do canal radicular e 
fechamento apical. Uma tomografia computadorizada de feixe cônico foi realizada durante o acompanhamento de dois anos e confirmou estes achados, porém não mostrou calcificação completa do canal radicular. Este estudo concluiu que nesta nova proposta de terapia de revascularização pode ser utilizado o gel de clorexidina à $2 \%$ para o tratamento de canais radiculares imaturos necróticos.

Seghi et al. (2013) através de uma revisão bibliográfica intitulada Root Fortification, se propuseram a descrever os métodos restauradores que são utilizados para reforçar raízes de dentes que possuem formação incompleta, com paredes dentinárias finas e com uma maior incidência de fratura, que reduz o prognóstico geral além longo prazo. As considerações biomecânicas de reforço de raiz enfraquecidas também são discutidas nesse trabalho, e as informações mais atualizadas sobre análise de falhas, características da dentina natural e testes in vitro foram considerados. À luz destas considerações adicionais, os autores propuseram algumas recomendações, como a utilização de procedimentos adesivos, em casos de dentes imaturos.

Torabinejad et al. (2014) realizaram um estudo histológico, piloto animal, examinando dois dentes tratados com dois scaffolds. Os autores tiveram como objetivo avaliar o uso de coágulos sanguíneos com Plasma Rico em Plaquetas (PRP) como scaffold em Endodontia. Para examinar os tecidos gerados dentro dos canais radiculares, os pesquisadores extirparam as polpas de 21 dentes caninos de 7 filhotes de furões. O sangramento foi estimulado a partir dos tecidos periapicais e um coágulo sanguíneo foi induzido no espaço do canal radicular, no nível da junção amelocementária em 12 dentes. O PRP foi preparado e inserido nos canais em 9 dentes. O acesso foi selado com MTA. Sete caninos não foram operados e serviram como controles. Após três meses, os dentes e seus tecidos adjacentes foram removidos para avaliação. Os tecidos encontrados nos canais dos dentes experimentais foram comparados com os de controle. Como resultado, quase todos os dentes mostraram a presença de tecido intracelular semelhante a um osso, mas nenhuma evidência de espessamento da parede dentinária ou estreitamento apical foi observado nos dentes experimentais. Os autores concluíram que, neste modelo experimental, o uso de PRP ou coágulos sanguíneos durante endodontia regenerativa leva à formação de tecido intracelular sem uma contínua maturação radicular.

Saoud et al. (2014) avaliaram o sucesso e tempo de resolução clínica dos sintomas de dentes permanentes imaturos com necrose pulpar e seus resultados radiográficos de interesse, como deposição de tecido duro dentro da raiz. O estudo foi realizado com 20 dentes tratados com o protocolo de tratamento de revascularização. Suas alterações clínicas e radiográficas foram coletadas em intervalos regulares durante 1 ano. $\mathrm{E}$ as alterações radiográficas foram quantificadas. Como resultado, todos os 20 dentes tratados sobreviveram durante o período de 12 meses, com acompanhamento mensal, além de terem atingido os critérios para o sucesso aos 12 meses. Os dentes apresentaram aumento estatisticamente significativo na largura e comprimento da raiz e diminuição do diâmetro, embora as alterações em muitos casos tenham sido pequenas (de tal forma que o significado clínico não é claro). A variação percentual dentro do caso no diâmetro apical, após 3 meses, foi de 16\% e aumentou para 79\% após 12 meses, com 55\% apresentando o fechamento apical completo. Com esses resultados obtidos, os autores concluíram que, apesar do sucesso clínico ter sido altamente previsível com este procedimento, clinicamente o espessamento e alongamento radiculares significativos são menos previsíveis após 1 ano de acompanhamento. O fechamento apical foi o achado radiográfico mais consistente.

Lin et al. (2014) apresentam um relato de caso de revascularização/revitalização fracassada, que poderia ser devido à desinfecção inadequada do canal radicular sem a remoção mecânica do biofilme e das bactérias nos túbulos dentinários. Um menino de 6 anos teve uma lesão traumática no dente, que foi avulsionado e replantado em 40 minutos. O dente subseqüentemente desenvolveu um inchaço local na área periapical. A paciente foi encaminhada à Clínica Endodôntica de PósGraduação para revascularização/revitalização do dente. O dente tratado permaneceu assintomático por 16 meses e depois desenvolveu dor e edema periapical local. O cirurgião dentista extraiu o dente revascularizado/revitalizado, que em seguida, foi processado para exame histológico e histobacteriológico. O tecido no canal foi completamente destruído. A maioria das 
bactérias foi observada na porção apical e não na porção coronal do canal e formou biofilme nas paredes do canal e penetrou nos túbulos dentinários. Com base nas observações histobacteriológicas, a falha do dente revascularizado/revitalizado pode ser devido à desinfecção inadequada do canal radicular sem desbridamento mecânico. Pode ser importante realizar o desbridamento mecânico como parte da terapia da revascularização/revitalização para romper o biofilme nas paredes do canal e remover as bactérias nos túbulos dentinários porque a terapia de revascularização/ revitalização é capaz de aumentar o espessamento das paredes do canal.

Chen et al. (2015) realizaram um estudo que questionava se a revascularização em dentes permanentes imaturos é uma técnica efetiva e reproduzível. O objetivo do trabalho foi rever casos clínicos de revascularização em seres humanos para avaliar a sua utilidade e reprodutibilidade. Como metodologia, uma pesquisa eletrônica estruturada de artigos científicos publicados entre 2001 e 2014 foi realizada com os seguintes termos: 'revascularização da polpa', 'revitalização da celulose' e/ou 'dente imaturo'. Os casos realizados em seres humanos foram selecionados, revisados e organizados em dois gráficos, incluindo informações do paciente, diagnóstico, tratamento e resultados em visitas de acompanhamento. Noventa e sete de 101 dentes (96,0\%) foi tratado com sucesso com a técnica de revascularização. A gama de variações técnicas disponíveis para irrigação, a desinfecção e a indução de coágulos sanguíneos têm um impacto insignificante na clínica. Durante as visitas de acompanhamento, o fechamento apical foi detectado em menos casos $(55,4 \%)$ em relação a outros fenômenos de apicigênese (aumento do comprimento da raiz, 76,2\%, aumento largura da raiz, 79,2\%). A revisão mostra que a revascularização demonstrou aumento acentuado no comprimento da raiz, largura e fechamento apical nos casos que foram relatados independentemente de variáveis clínicas.

Galler (2016) realizou uma revisão de literatura abordando os conhecimentos e considerações referentes aos procedimentos clínicos de revitalização ou regeneração pulpar em dentes com formação incompleta de raízes e polpas necrosadas. Idealmente os procedimentos regenerativos endodônticos não resolvem apenas a dor, inflamação ou lesão periapical, mas a formação de um tecido imunocompetente no interior do canal, que possa reconstituir a estrutura biológica original, a função da polpa dental e assim levar a um aumento em espessura e comprimento radicular e a força das paredes dentinárias previamente finas e propensas à fratura. Concluiu-se que, embora os relatos de casos existentes na literatura demonstrem resultados promissores, o protocolo para o tratamento endodôntico não está totalmente estabelecido em dentes imaturos com polpa necrosada. Questões em relação à terminologia, seleção e consentimento informado, bem como detalhes dos procedimentos, especialmente na escolha de irrigantes, medicamentos e materiais para selamento de cavidades, permanecem ainda variados e sem o protocolo consensual.

Conde et al. (2016) publicaram o estudo em formato de revisão avaliando os aspectos relevantes para o sucesso clínico e formação tecidual em casos de revascularização pulpar. Avaliaram a taxa de sobrevivência e a natureza do tecido formado dentro dos canais radiculares dos dentes permanentes imaturos necróticos humanos (NIPT) sob revascularização do canal radicular. A pesquisa foi realizada no SciVerse Scopus ${ }^{\circledR}$, PubMed / Medline, Web of Science®, BIREME e em literatura. Os pesquisadores constataram que um total de 367 NIPT foram submetidos à revascularização, dos quais apenas 21 necessitaram de tratamento endodôntico. O tecido novo era cemento e osso pobremente mineralizado positivo à Sialoproteína Óssea, mas negativo à Sialoproteína Dentinária. As falhas foram associadas principalmente à reinfecção do canal radicular. A maioria dos estudos incluídos relatou um aumento significativo tanto no comprimento quanto na largura da raiz. No entanto, uma vez que a maioria destes dados veio de relatos de casos, eles devem ser interpretados com cuidado, já que a maioria dos relatos de casos foi focada no sucesso do tratamento (e não falhas). Portanto, Conde e seus colaboradores concluíram que ensaios clínicos randomizados e mais bem desenhados que comparam a revascularização pulpar com tratamentos de apicificação disponíveis são necessários para resolver esta lacuna na literatura. 
Verma et al. (2016) evidenciaram através de estudo in vivo, a importância da desinfecção do canal radicular, eliminando bactérias residuais, para o sucesso dos casos de regeneração pulpar. O objetivo do estudo foi determinar radiograficamente e histologicamente o efeito de bactérias residuais sobre o resultado da regeneração da polpa mediada por uma formação de tecido através de células-tronco da polpa em comparação com a revascularização tradicional. Como metodologia, os autores utilizaram 24 dentes caninos de 6 furões e induziram lesões periapicais. Após desinfecção com $\mathrm{NaOCl}$ a 1,25\% e pasta triantibiótica, as células-tronco da polpa dentária de furão, encapsuladas num Scaffold de hidrogel, foram injetadas em metade dos dentes experimentais. A outra metade foi tratada com o protocolo tradicional de revascularização, com a indução de coágulo sanguíneo. Após 3 meses, os dentes caninos foram radiografados e processados para análise histológica e histobacteriológicas. Os pesquisadores trouxeram como resultados a afirmação de que não houve diferença significativa entre os dois grupos experimentais no desenvolvimento radicular radiográfico. A presença de bactérias residuais foi significativamente associada à falta de crescimento radiográfico. A quantidade de tecido mineralizado associado à dentina formada nos dentes com bactérias residuais foi significativamente menor do que em dentes sem bactérias residuais. A partir dos resultados obtidos, pôde-se concluir que as bactérias residuais têm um efeito negativo no tratamento endodôntico regenerativo.

Moradi et al. (2016) avaliaram o efeito indutivo do plasma rico em plaquetas (PRP) na expressão de fatores da angiogênese e revascularização pulpar de dentes necróticos imaturos. Neste estudo animal experimental, os autores dividiram aleatoriamente 28 pré-molares imaturos de cães de raça mista em quatro grupos, dois experimentais negativos e um controle positivo. Os pré-molares no grupo de controle negativo foram deixados intactos para se desenvolverem normalmente. Nos grupos controle positivo e experimental, foram removidas as polpas, induzida a necrose pulpar e, logo após, as câmaras foram seladas. Em seguida, aplicaram o protocolo de revascularização nos dentes experimentais localizados no quadrante direito. Dois meses após, o mesmo protocolo foi aplicado ao quadrante esquerdo. Os canais radiculares foram desinfetados por irrigação com solução de hipoclorito de sódio $(\mathrm{NaOCl})$ e aplicação de uma pasta antibiótica tripla. Após a indução de um coágulo sanguíneo (blood clot-BC) no interior do espaço do canal, à porção coronária dos canais foi atribuído qualquer um dos dois grupos experimentais: grupo 1 [BC + PRP + MTA] e grupo $2(\mathrm{BC}+\mathrm{MTA})$. As mandíbulas que mantinham os dentes foram processadas para análise histológica de tecido recém-formado e avaliação imunohistoquímica, de acordo com o fator de crescimento do endotélio vascular (VEGF) e expressões do fator VIII nos canais. Como resultado, os pesquisadores observaram que a análise histológica não demonstrou diferença significativa na formação de novo tecido vital dentro dos canais radiculares entre os grupos 1 e 2. Com os resultados obtidos, conclui-se que o PRP não pôde aumentar a formação de novo tecido vital. Na imunohistoquímica, os resultados mostraram que o VEGF e o fator VIII desempenharam uma formação de novos vasos dentro dos canais radiculares de dentes imaturos necrosados.

Aldakak et al. (2016) relataram um caso clínico de uma paciente do sexo feminino de 11 anos que foi encaminhada tendo como queixa principal dor no segundo pré-molar mandibular direito. Os exames clínicos e radiográficos apresentaram cáries secundárias sob uma antiga restauração composta, resposta de sensibilidade pulpar negativa e raiz imatura com ápice aberto. Após a limpeza e modelagem do canal radicular, o coágulo sangüíneo foi induzido no interior do canal até $2 \mathrm{~mm}$ abaixo da junção cemento-esmalte. Após 10 minutos para permitir a coagulação a este nível, colocou-se um tampão de Biodentine sobre o coágulo sanguíneo e o dente foi selado com cimento de ionômero de vidro. Na próxima visita, o dente estava livre de sintomas e uma restauração permanente foi realizada. Os exames clínicos e radiográficos durante um acompanhamento de dois anos mostraram maturação completa das raízes e tecidos moles de suporte intactos sem trato sinusal, dor ou inchaço. Os autores tomaram como conclusão que o uso de Biodentine em um protocolo de uma única visita para o tratamento de um dente permanente imaturo com polpa necrótica pode criar um ambiente adequado para revascularização, resultando na maturação da raiz. 
Silujjai e Linsuwanont (2017) realizaram um estudo comparando os resultados clínicos e radiográficos da apicificação com o MTA e a revascularização em dentes permanentes imaturos não vivos, e analisaram os fatores que influenciaram o resultado do tratamento. Quarenta e seis casos (29 casos de apicificação e 17 casos de revascularização) foram escolhidos para este estudo. As informações pré-operatórias e pós-operatórias dos pacientes foram analisadas. Os resultados do tratamento foram categorizados como um sucesso ou fracasso e retenção funcional. O desenvolvimento adicional da raiz foi avaliado em termos de porcentagem no comprimento da raiz e largura da raiz. Como resultados, os autores confirmaram que as taxas de sucesso da apicificação com MTA e revascularização foram de $80,77 \%$ e $76,47 \%$ e a retenção funcional foi de $82,76 \%$ e $88,24 \%$, respectivamente. A revascularização proporcionou maior porcentagem de mudanças na largura da raiz (13,75\%) em comparação com o MTA $(3,30 \%)$. A porcentagem média de alteração do aumento do comprimento da raiz foi de $9,51 \%$ no grupo da revascularização e 8,55\% no grupo da apicificação com MTA. A revascularização mostrou graus de comprimento de raiz aumentado variando de $4 \%$ a 58\%. A fratura foi a principal causa de falha nos dentes com apicificação com MTA. Com isso, os pesquisadores concluíram que tanto a apicificação com MTA quanto a revascularização proporcionam um resultado confiável no aspecto de resolução da doença, porém nenhum destes tratamentos apresenta um desenvolvimento radicular adicional previsível.

He et al. (2017) publicaram um estudo questionando sobre o conceito de endodontia regenerativa, a possibilidade de ser estendido para revitalizar dentes permanentes maduros com diagnósticos incluindo pulpite irreversível e/ou necrose pulpar em adultos. $\mathrm{O}$ trabalho traz como objetivo descrever não apenas as descobertas emergentes para revitalizar dentes permanentes maduros em pacientes adultos, mas também identificar desafios e estratégias que focalizam a realização do objetivo da endodontia regenerativa em adultos, apresentando casos clínicos e descrevendo a base biológica de potenciais procedimentos endodônticos regenerativos em adultos.

Timmerman e Parashos (2018) escreveram um relato de caso de procedimento regenerativo em um dente o qual sofreu como consequência a descoloração coronária, e submetido a clareamento dentário interno. Uma tomografia computadorizada de feixe cônico foi realizada para investigar a formação de tecidos duros dentro do canal radicular, bem como a formação de tecido calcificado. Cinco anos após o REP com MTA houve completa cicatrização periapical, formação de tecido duro dentro do canal radicular e completa maturação do ápice radicular, uma ponte de tecido duro foi notada na junção cemento-esmalte quando a câmara pulpar. O elemento dentário foi submetido a clareamento interno e a cor normal foi obtida após semanas de clareamento interno. Nesse caso foi utilizado MTA e uma matriz de celulose que demonstrou não impedir a regeneração do tecido e ser reabsorvida por estes tecidos. Outros materiais com estabilidade de cor devem ser selecionados como barreiras para REPs, para evitar um potencial efeito adverso do MTA no desfecho e descoloração do REP.

Montero-Miralles et al. (2018) com o objetivo de analisar o uso de antibióticos em REPs, realizaram uma revisão de literatura científica sobre: (i) Eficácia de antibióticos usados em REPs contra bactérias implicadas na doença endodôntica; (ii) evidência científica que apoia o uso de antibióticos tópicos em REPs; (iii) implicações clínicas do uso de antibióticos em REPs e os possíveis efeitos colaterais; (iv) Efeito dos antibióticos nas células estaminais da polpa dentária; e (v) pesquisa em andamento sobre o uso de antibióticos em REPs. Antibióticos utilizados em REPs são eficazes contra bactérias implicadas em infecções endodônticas. As pastas antibióticas triplas com minociclina atingem a desinfecção completa dos dentes imaturos com polpas necróticas, sem afetar o SCAP. Estudos experimentais realizados em cães apoiam o uso de antibióticos em REPs. Estudos clínicos relatam altas taxas de sucesso de REPs usando antibióticos como curativos intracanal. No entanto, a descoloração dentária é um importante efeito colateral do uso da pasta tripla antibiótica. Uma pasta antibiótica contendo apenas metronidazol e ciprofloxacina pode ser uma boa alternativa. O uso de scaffolds contendo antibióticos ou polímero de antibiótico triplo modificado por clindamicina (metronidazol, ciprofloxacina e clindamicina) poderia ser um sistema de liberação de drogas antimicrobianas biologicamente seguras em REPs. 
No mesmo ano, Eramo et al. (2018) realizaram uma revisão sistemática examinando a técnica de cell homing para a regeneração da polpa dentária, selecionando artigos sobre experimentos in vitro, modelos de transplante ectópico in vivo e revascularização da polpa in situ. As bases de dados MEDLINE / PubMed e Scopus foram pesquisadas eletronicamente para artigos sem limites na data de publicação. Dois revisores examinaram e incluíram artigos de forma independente, de acordo com os critérios de seleção predefinidos. As buscas eletrônicas identificaram 46 estudos. Após o título, resumo e exame de texto completo, 10 artigos preencheram os critérios de inclusão. Dados in vitro destacaram que múltiplas citocinas têm a capacidade de induzir migração, proliferação e diferenciação de células-tronco / progenitoras da polpa dentária. A maioria dos estudos in vivo obteve tecidos regenerados semelhantes a polpa com neovascularização. Em alguns casos, as amostras mostraram nova inervação e nova deposição de dentina. A revascularização da polpa in situ regenerou tecidos semelhantes a polpa intracanal com neovascularização, inervação e formação de dentina. As estratégias de orientação celular para a regeneração da polpa precisam de maior compreensão e aprimoramento para que se tornem uma abordagem confiável e eficaz na endodontia. No entanto, o cell homing representa a fonte clinicamente viável para a regeneração da polpa dentária.

Nosrat et al. (2019) realizaram um estudo, pelo qual examinaram clínica, radiograficamente e histologicamente o resultado dos tratamentos endodônticos regenerativos (TER) em dentes humanos não infectados e imaturos usando o SynOss Putty como suporte. Foram incluídos três pares de primeiros pré-molares maxilares / mandibulares em três pacientes programados para extração. Testes de sensibilidade confirmaram a presença de polpas vitais. Após consentimento informado, anestesia e isolamento absoluto, as polpas foram removidas. O TER foi realizado usando os seguintes suportes: SynOss Putty + sangue em ambos os dentes no paciente \# 1, SynOss Putty com ou sem sangue no paciente \# 2, e SynOss Putty + sangue ou sangue somente no paciente \# 3. Após um período de acompanhamento de 2,5 a 7,5 meses, os dentes foram avaliados clínica e radiograficamente, extraídos e examinados histologicamente. Após o tratamento, os pacientes permaneceram assintomáticos. $\mathrm{O}$ exame radiográfico dos dentes mostrou sinais de desenvolvimento radicular após o tratamento. Em dentes tratados com SynOss Putty + sangue, o exame histológico mostrou a formação de tecido mineralizado intracanal ao redor das partículas do scaffold, solidificando-se com tecido neoformado cimentado nas paredes dentinárias. O dente tratado com SynOss Putty sem sangue mostrou a formação de uma lesão periapical. $\mathrm{O}$ dente tratado com um coágulo só mostrou tecidos de origem periodontal crescendo no espaço do canal radicular. Os autores concluíram que SynOss Putty + Blood mostrou um padrão previsível de formação de tecido e mineralização quando utilizado como suporte para RET em dentes humanos não infectados e imaturos. O tecido mineralizado recém-formado solidifica com o cemento recém-formado nas paredes dentinárias.

Simay e Massimo (2020), realizaram uma revisão sistemática e de meta-análise com o objetivo de avaliar se há uma correlação entre a etiologia da necrose pulpar e o sucesso do tratamento endodôntico regenerativo (TER). Foram realizadas pesquisas eletrônicas nas bases de dados: PubMed, Scopus, Web of Science, Cochrane Central Register of Controlled Trials, Embase. Foram incluídos estudos de tratamento endodôntico regenerativo em que houveram avaliação clinica e radiográfica com no mínimo de 6 meses de acompanhamento, para avaliar a maturação radicular, assim como estudos que especificavam as causas da necrose pulpar. Somado ao questionamento inicial do estudo acerca da influência etiológica da necrose $\mathrm{x}$ sucesso do TER, outros fatores foram avaliados como o tipo do dente, medicação intracanal, protocolo de irrigação, uso da matriz de colágeno, e o tipo de scaffold foram avaliados para uma possível relação com o resultado. Utilizando estudos randomizados e não-randomizados, foram obtidos como resultado 1197 itens, que após triagem, incluíram-se 18 estudos contendo 445 casos de TER. A taxa de sucesso para 274 dentes com etiologia de trauma foi de 94,8\% para trauma, 95 dentes com etiologia de dens invaginatus foi de $93,1 \%$, e para 24 dentes com cárie de $96 \%$. Nenhuma diferença significativa foi encontrada entre os resultados de TER entre dentes traumatizados, com cárie e dens invaginatus (P 5.055). Estudos de meta-análise comparando dentes com cárie vs dens invaginatus, dentes com cárie vs trauma, confirmaram que não houve evidência de diferença nos resultados. 
Wikström et al. (2021), realizaram uma revisão sistemática com o objetivo de avaliar os conhecimentos atuais sobre técnicas de apicificação e técnicas regenerativas, como modalidades de tratamento e com finalidade de mapear evidências científicas para a eficácia de ambos os métodos de manejo de dentes imaturos traumatizados com necrose pulpar e periodontite apical. Para este estudo, as bases de dados utilizadas foram: PubMed, Web of Science, Cochrane Library, Ovid (Medline) e Embase. Com as seguintes palavras chaves: Regenerative endodontic treatment, regenerat, revital, endodontic regeneration, regenerative endodontics, pulp revascularization, revasculari, traumatized immature teeth. Apenas estudos revisados em pares com 20 casos acompanhados por 24 meses foram incluídos, a avaliação da elegibilidade foi realizada de forma independente e cega por três revisores, sendo as discordâncias resolvidas por um consenso. Foram avaliados três resultados: sobrevivência, sucesso e desenvolvimento da raiz. Uma análise qualitativa revelou que tanto a TER quanto a apicificação tiveram taxas iguais de sucesso e sobrevivência, e se mostraram eficazes no tratamento de dentes permanentes imaturos com necrose pulpar. As TERs parecem ser superiores às técnicas de apicificação em termos de estimulação da maturação radicular, ou seja, o espessamento da parede da raiz e alongamento da mesma. Foram identificadas lacunas de conhecimento em relação ao tratamento e protocolos de acompanhamento em ambas as técnicas.

\section{Discussão}

A revascularização pulpar é uma técnica alternativa promissora em casos de dentes permanentes com ápices imaturos necrosados, que promove a formação de tecido dentário em comprimento e espessura da raiz, bem como redução do diâmetro apical, porém sem resultados previsíveis (Banchs \& Trope, 2004; Jung et al., 2008; Shah et al., 2008; Ding et al., 2009; Cehreli et al., 2011; Wigler et al., 2013; Chen et al., 2015; He et al., 2017; Wikström et al., 2021 ).

E na maioria dos casos, há a resolução completa dos sinais e sintomas clínicos e a cicatrização de lesões periapicais ( Shah et al., 2008; Galler, 2016).

O maior desafio da revascularização pulpar é o controle da infecção, pois os fracassos associados à técnica estão diretamente relacionados à presença de bactérias residuais localizadas nos túbulos dentinários, principalmente na porção coronária radicular (Lin $e t$ al., 2014; Verma et al., 2016). Um dos passos do tratamento regenerativo tem como compromisso promover uma desinfecção suficiente com um mínimo de danos às estruturas celulares (Galler, 2016).

Um protocolo para a revascularização pulpar foi descrito por AAE e baseia-se na seguinte sequência: descontaminação do canal radicular com mínimo de desgaste das paredes; medicação intracanal por 21 dias estimando retorno sem sinais ou sintomas; remoção da medicação em segunda sessão; irrigação com $20 \mathrm{~mL}$ de EDTA 17\%; indução de sangramento via periápice através de transpasse de lima manual $2 \mathrm{~mm}$ além do comprimento do dente, ou inserção de PRF, ou matriz de fibrina autóloga; proteção do tecido através de selamento dos 3 a $4 \mathrm{~mm}$ cervicais da raiz com material biocompatível e alta capacidade seladora, evitando microinfiltraçao bacteriana (American Association of Endodontists, 2016).

A grande maioria dos autores utilizam o $\mathrm{NaOCl}$ como solução irrigadora auxiliar durante a revascularização pulpar ( Banchs \& Trope, 2004 ; Jung et al., 2008; Shin et al., 2009; Cehreli et al., 2011; Chen et al., 2015; Conde et al., 2016; Moradi et al., 2016; Verma et al., 2016). As concentrações variam entre 1\% (Timmerman \& Parashos, 2018), 1,25\% (Verma et al., 2016), 2,5\% (Shah et al., 2008; Cehreli et al., 2011; Verma et al., 2016), 5,25\% (Banchs \& Trope, 2004; Ding et al., 2009; Lin et al., 2014; Aldakak et al., 2016; Moradi et al., 2016) e até 6\% (Shin \& Albert, 2009). É também frequentemente associado principalmente à clorexidina em concentrações entre 0,12\% (Banchs \& Trope, 2004; Saoud et al., 2014) a 2\% (Shin \& Albert, 2009; Aldakak et al., 2016). Um único trabalho utilizou somente digluconato de clorexidina a $2 \%$ como solução irrigante durante o tratamento (Soares et al., 2013).

A partir de um estudo in vitro realizado pôde-se observar que a irrigação com uma concentração alta de $\mathrm{NaOCl}$ tem um efeito negativo severo na sobrevivência e diferenciação das células-tronco da papila apical. Sendo sugerida a utilização de 
irrigação em baixa concentração de $\mathrm{NaOCl}$ (1,5\%) (Martin et al., 2014; Koç \& Del Fabbro, 2020) seguida por irrigação de EDTA (17\%) para minimizar os riscos de efeito citotóxicos do $\mathrm{NaOCl}$ nas células-tronco, assim como possibilitar a indução de moléculas sinalizadoras dentinárias (Galler et al., 2011; Koç \& Del Fabbro, 2020).

É questionável que o atual protocolo de desinfecção radicular dos procedimentos de revascularização/revitalização que não possuem desbridamento mecânico do biofilme nas paredes do canal seja capaz de eliminar efetivamente a maioria das bactérias in vivo (Lin et al., 2014). Seguindo este pensamento, em alguns trabalhos houve a adição de uma leve instrumentação das paredes dos canais visando à remoção da polpa necrótica e desorganização do biofilme intrarradicular (Jung et al., 2008; Jadhav et al., 2012; Soares et al., 2013; Aldakak et al., 2016; Verma et al., 2016).

Grande parte dos trabalhos descritos utilizaram, como medicação intracanal, a pasta triantibiótica, composta por metronidazol, ciprofloxacina e minociclina por ser um potencial agente contra patógenos (Banchs \& Trope, 2004; Jung et al., 2008; Shah et al., 2008; Ding et al., 2009; Saoud et al., 2014; Moradi et al., 2016; Verma et al., 2016). O escurecimento dentinário está associado à pasta devido à presença de minociclina em sua composição, podendo ser substituída por clindamicina (Montero-Miralles et al., 2018), cefaclor (Wigler et al., 2013; Montero-Miralles et al., 2018) ou apenas removida da composição da pasta (Wigler et al., 2013; Montero-Miralles et al., 2018). Outros dois fatores importantes a serem levados em consideração são o risco de seleção bacteriana e reações alérgicas relacionadas ao uso desta pasta (Soares et al., 2013; Wigler et al., 2013; Montero-Miralles et al., 2018).

Considerando estas desvantagens, alguns trabalhos utilizaram como medicação intracanal o hidróxido de cálcio, já que o mesmo possui atividade antimicrobiana e libera fatores de crescimento e biomoléculas de dentina, podendo ser adicionado a veículos inertes restritos a porção cervical da raiz (Cehreli et al., 2011) ou ativo como o digluconato de clorexidina a $2 \%$ adicionado na porção média e cervical da raiz (Soares et al., 2013). Embora apresente todas essas propriedades, o uso de hidróxido de cálcio é considerado prejudicial à revascularização pulpar, já que apresenta pH elevado podendo causar necrose por contato de tecidos com potencial de diferenciação adicionado ao risco de fratura a longo prazo (Banchs \& Trope, 2004; Seghi et al., 2013). Mesmo com divergência entre autores e protocolos clínicos, ambos os medicamentos utilizados mostram-se eficientes na resolução da infecção intracanal.

A indução de um coágulo sanguíneo é o scaffold escolhido, pela grande maioria dos autores, para funcionar como meio de crescimento e diferenciação de células tronco mesenquimais no interior do canal radicular em dentes imaturos necrosados (Banchs \& Trope, 2004; Shah et al., 2008; Ding et al., 2009; Shin et al., 2009; Soares et al., 2013; Lin et al. 2014; Saoud et al., 2014; Aldakak et al., 2016; Galler, 2016; Eramo, et al., 2018). A utilização de PRP pode melhorar o resultado biológico desejado do processo endodôntico regenerativo, mas quando comparado ao uso do coágulo sanguíneo não obteve resultados estatisticamente diferentes no que se trata de desenvolvimento radicular (Jadhav et al., 2012; Torabinejad et al., 2014; Moradi et al., 2016; Verma et al., 2016). Outro tipo de scaffold encontrado na literatura é o SynOss Putty com sangue, que pode tornar o resultado dos tratamentos endodônticos regenerativos mais previsível (Nosrat et al., 2019).

O MTA foi o material de selamento radicular cervical utilizado praticamente em todos os casos de revascularização pulpar devido ao selamento cervical efetivo após a formação de coágulo sanguíneo, além de ser um excelente material antimicrobiano (Banchs \& Trope, 2004; Jung et al., 2008; Ding et al., 2009; Shin \& Albert, 2009; Cehreli et al., 2011; Soares et al., 2013; Lin et al., 2014; Saoud et al., 2014; Moradi et al., 2016; Verma et al., 2016) , mas que pode estar associado ao escurecimento dentário (Timmerman \& Parashos, 2018). Biodentine (Septodont, Pomerode/SC, Brasil) também é um excelente material de escolha (Aldakak et al., 2016), não havendo diferença na proposta final, dentre os materiais, para revascularização pulpar.

$\mathrm{Na}$ literatura podemos encontrar casos clínicos de revascularização pulpar em uma única sessão clínica. São citadas algumas vantagens para esse tipo de abordagem: redução das chances de contaminação bacteriana 
adicional intracanal, além de que um único protocolo de revascularização pode superar o problema da baixa adesão do paciente às visitas de acompanhamento, reduzindo o número de consultas necessárias e, por último, torna-se uma técnica mais conservadora, concentrando-se na preservação do tecido pulpar vital remanescente e célulastronco mesenquimais da papila apical (Shin et al., 2009; Aldakak et al., 2016; Timmerman \& Parashos, 2018).

Ao avaliar o desfecho do procedimento, vários artigos examinaram a existência de aumento significativo em espessura dentinária e comprimento radicular após o processo de revascularização pulpar (Jung et al., 2008; Shah et al., 2008; Ding et al., 2009; Cehreli et al., 2011; Jadhav et al., 2012; Soares et al., 2013; Saoud et al., 2014; Silujjai \& Linswanont, 2017; Conde et al., 2018; Wisktrom et al., 2020). Histologicamente, o trabalho de Torabinejad e colaboradores concluiu que mesmo com a presença de um tecido intracelular semelhante a um osso, nenhuma evidência de espessamento da parede dentinária ou estreitamento apical foi observado (Torabinejad et al., 2014).

Alguns autores afirmam a necessidade de uma avaliação imaginológica padronizada para realizar o acompanhamento e evolução do procedimento (Cehreli et al., 2011; Soares et al., 2013), mesmo sendo a definição de parâmetros para comparação difícil (Conde et al., 2018). O período ideal para a retorno do paciente é de 3, 6, 12, 18 e 24 meses, depois disso anualmente pelos próximos 5 anos (Wigler et al., 2017).

Apesar dos resultados promissores observados em várias séries de casos publicados (Shah et al., 2008; Ding et al., 2009; Cehreli et al., 2011; Jadhav et al., 2012; Saoud et al., 2014; Silujjai et al., 2017; Wigler et al., 2017), o processo de revascularização pulpar não é uma terapêutica estabelecida devido à falta de ensaios clínicos bem delineados que avalie o prognóstico (Galler, 2016). A impossibilidade de induzir o sangramento inicial compreende uma das principais causas de falha na revascularização pulpar (Ding et al., 2009). Em dentes com múltiplas raízes, após observando um nível inadequado de hemorragia em alguns canais, transferindo sangue entre os canais constituiu uma estratégia eficaz, como realizado por Cehreli e colaboradores (Cehreli et al., 2011). A falta de desbridamento mecânico também está associada a falhas nos procedimentos regenerativos (Lin et al., 2014 Galler, 2016). O escurecimento dentinário associado à medicação intracanal e aos materiais seladores pode ser resolvido através de clareamento interno após a resolução do processo de regeneração (Timmerman \& Parashos, 2018). No que se refere à reabilitação funcional do dente, faz sentido fortalecer a raiz com um método restaurador que é durável, na tentativa de minimizar as deformações cíclicas que podem ocorrer no dente sob a função normal (Seghi et al., 2013).

\section{Considerações Finais}

Os protocolos atuais de técnica de revascularização pulpar para o tratamento de dentes permanentes imaturos com polpa irreversivelmente danificada devem ser considerados como opções de tratamento valiosas, eficazes e reprodutíveis na clínica. Com base nos artigos selecionados para esta revisão, a técnica de revascularização mostrou aumento acentuado no comprimento, largura e fechamento apical dos casos relatados. Essa técnica, independentemente da metodologia individual, pode aumentar o desenvolvimento da raiz em casos selecionados corretamente, tendo vantagens potenciais em relação a outras alternativas disponíveis. No entanto, para um melhor conhecimento sobre a taxa real de sucesso dessa técnica, faz-se necessário mais estudos randomizados e com controle de avaliação de imagens radiográficas durante visitas de acompanhamento. Embora o reparo, em vez da regeneração real, seja alcançado com os protocolos atuais, espera-se que pesquisas adicionais na área sejam realizadas na área de engenharia tecidual baseada em células-tronco, para que permitam a verdadeira regeneração e melhores resultados de tratamento de maneira viável para aplicação clínica. 


\section{Referências}

Al Ansary, M. A. D., Day, P. F., Duggal, M. S., \& Brunton, P. A. (2009). Interventions for treating traumatized necrotic immature permanent anterior teeth: inducing a calcific barrier \& root strengthening. Dental Traumatology. 25, 367-379.

American Association of Endodontists (AAE). (2016). Clinical Considerations for a Regenerative Procedure [acesso em outubro, 2017]. Disponível em http://www.aae.org/uploadedfiles/publications_and_research/research/currentregener ativeendodonticconsiderations.pdf.

Aldakak, M. M. N., Capar, I. D., Rekab, M. S., \& Abboud, S. (2016). Single-Visit Pulp Revascularization of Nonvital Immature Permanent Tooth Using Biodentine. Iranian Endodontic Journal. 11 (3), 246-249.

Banchs, F., \& Trope, M. (2004). Revascularization of immature permanent teeth with apical periodontitis: new treatment protocol? Journal of Endodontics. 30(4), 196-200.

Botero, T. M., Tang, X., Gardner, R., Hu, J. C. C., Boynton, J. R., \& Holland, G. R. (2017). Clinical Evidence for Regenerative Endodontic Procedures: Immediate versus Delayed Induction?. Journal of Endodontics. 43, 75-81.

Cehreli, Z. C., Isbitiren, B., Sara, S., \& Erbas, G. (2011). Regenerative Endodontic Treatment (Revascularization) of Immature Necrotic Molars Medicated with Calcium Hydroxide: A Case Series. Journal of Endodontics. 37(9), 1327-1330.

Conde, M. C. M., Chisini, L.A., Sarkis-Onofre, R., Schuch, H. S., Nör, J. E., \& Demarco, F. F. (2016) A Scoping review of Root Canal Revascularization: Relevant Aspects for Clinical Success and Tissue Formation. International Endodontic Journal. 50(9), 860-874.

Chen, Y., Jovani-Sancho, M. D. M., \& Sheth, C. C. (2015). Is revascularization of immature permanent teeth an effective and reproducible technique?. Dental Traumatology. 31, 429-436.

Ding, R. Y., Cheung, S., Chen, J., Yin, X. Y., Wang, Q. Q., \& Zhang, C. F. (2009). Pulp Revascularization of Immature Teeth With Apical Periodontitis: A Clinical Study. Journal of Endodontics. 35(5), 745-749.

Eramo, S., Natali, A., Pinna, R., \& Milia, E. (2018). Dental pulp regeneration via cell homing. International Journal Endodontic. 51(4), 405-419.

Galler, K. M., D’Souza, R. N., Federlin, M., Cavender, A. C., Hartgenrink, J. D., Hecker, S., \& Schmalz, G. (2011). Dentin conditioning codetermines cell fate in regenerative endodontics. J Endod. 37, 1536-41.

Galler, K. M. (2016). Clinical Procedures for Revitalization: Current Knowledge and Considerations. International Endodontic Journal. 49 , $926-936$.

He, L., Kim, S. G., Gong, Q., Zhong, J., Wang, S., Zhou, X., Ye, L., Ling, J., \& Mao, J. J. (2017). Regenerative endodontics for adults patients. Journal of Endodontics. 43 (9S), 57-64.

Jadhav, G., Shah, N., \& Logani, A. (2012). Revascularization with and without Platelet-rich Plasma in Nonvital, Immature, Anterior Teeth: A Pilot Clinical Study. Journal of Endodontics. 38(12), 1581-1587

Jeeruphan, T., Jantarat, J., Yanpiset, K., Suwannapan, L., Khewsawai, P., Hargreaves, K. M. (2012). Mahidol study 1: comparison of radiographic and survival outcomes of immature teeth treated with either regenerative endodontic or apexification methods: a retrospective study. Journal of Endodontics. 38(10), 13306 .

JO, Farik, B., \& Munksgaard, E. C. (2002). Long-term calcium hydroxide as a root canal dressing may increase risk of root fracture. Dental Traumatology. 18, 134-137.

Jung, I. Y., Lee, S. J., \& Hargreaves, K. M. (2008). Biologically Based Treatment of Immature Permanent Teeth with Pulpal Necrosis: A Case Series. J Endod. 34(7), 876-887.

Koç, S., \& Del Fabbro, M. (2020). Does the Etiology of Pulp Necrosis Affect Regenerative Endodontic Treatment Outcomes? A Systematic Review and Metaanalyses. J Evid Based Dent Pract. 20(1),101-400.

Lin, L. M., Shimizu, E., Gibbs, J. L., Loghin, S., \& Ricucci, D. (2014). Histologic and histobacteriologic observations of failed revascularization/revitalization therapy: a case report. Journal of Endodontics. 40(2), 291-5.

Martin, D. E., De Almeida, J. F. A, Henry, M. A., Teixeira, F. B., \& Diogenes, A. (2014). Concentrationdependent effect of sodium hypochlorite on stem cells of apical papilla survival and differentiation. J Endod. 40, 51-5.

Montero-Miralles, P., Martín-González, J., Alonso-Ezpeleta, O., Jiménez-Sánchez, M. C., Velasco-Ortega, E., \& Segura-Egea, J. J. (2018). Effectiveness and clinical implications of the use of topical antibiotics in regenerative endodontic procedures: a review. International Journal Endodontic. 51(9), 981-988.

Moradi, S., Talati, A., Forghani, M., Jafarian, A. H., Naseri, M., \& Shojaeian, S. H. (2016). Immunohistological evaluation of revascularized immature permanent necrotic teeth treated by platelet-rich plasma: an animal investigation. Cell Journal. 18(3), 389-396.

Nosrat, A., Kolahdouzan, A., Khatibi, A. H., Verma, P., Jamshidi, D., Nevins, A. J., \& Torabinejad, M. (2019). Clinical, radiographic and histologic outcome of regenerartive treatment in human teeth using a novel collagen-hydroxyapatite scaffolds. Journal of Endodontics. 45:13-143.

Saoud, T. M. A, Zaazou, A., Nabil, A., Moussa, S., Lin, L. M., \& Gibbs, J. L. (2014). Clinical and Radiographic Outcomes of Traumatized Immature Permanent Necrotic Teeth after Revascularization/Revitalization Therapy. Journal of Endodontics. 40(12), $1946-1952$.

Soares, A. J., Lins, F. F., Nagata, J. Y., Gomes, B. P. F. A., Zaia, A. A., Ferraz, C. C. R., et al. (2013). Pulp Revascularization after Root Canal Decontamination with Calcium Hydroxide and 2\% Chlorhexidine Gel. Journal of Endodontics. 39(3), 417-420. 
Research, Society and Development, v. 11, n. 2, e14111225546, 2022

(CC BY 4.0) | ISSN 2525-3409 | DOI: http://dx.doi.org/10.33448/rsd-v11i2.25546

Seghi, R. R., Nasrin, S., Draney, J., \& Katsube, N. (2013) Root Fortification. Journal of Endodontics. 39(3S), S57-S62.

Silujjai, J., \& Linswanont, P. (2017). Treatment Outcomes of Apexification or Revascularization in Nonvital Immature Permanent Teeth: A Retrospective Study. Journal of Endodontics. 43(2), 238-245.

Shah, N., Logani, A., Bhaskar, U., \& Aggarwal, V. (2008). Efficacy of Revascularization to Induce Apexification/Apexogensis in Infected, Nonvital, Immature Teeth: A Pilot Clinical Study. Journal of Endodontics. 34(8), 919-925.

Shin, S.Y., Albert, J. S., \& Mortman, R. E. (2009). One step pulp revascularization treatment of an immature permanent tooth with chronic apical abscess: a case report. International Endodontic Journal. 42, 1118-1126.

Timmerman, A., \& Parashos, P. (2018). Bleaching of a Discolored Tooth with Retrieval of Remnants after Successful Regenerative Endodontics. Journal of Endodontics. 44(1), 93- 97

Torabinejad, M., Faras, H., Corr, R., Wright, K. R., \& Shabahang, S. (2014). Histologic Examinations of Teeth Treated with 2 Scaffolds: A Pilot Animal Investigation. Journal of Endodontics. 40(4), 515-520.

Verma, P., Nosrat, A., Kim, J. R., Price, J. B., Wang, P., Bair, E., et al. (2016). Effect of Residual Bacteria on the Outcome of Pulp Regeneration In Vivo. Journal of Dental Research. 96(1), 100-106.

Wigler, R., Kaufman, A. Y., Lin, S., Steinbock, N., Molina-Hazan, H., \& Torneck, C. D. (2013). Revascularization: A Treatment for Permanent Teeth with Necrotic Pulp and Incomplete Root Development. Journal of Endodontics. 39(3), 319-326.

Wikström, A., Brundin, M., Lopes, M. F., El Sayed, M., \& Tsilingaridis, G (2021). What is the best long-term treatment modality for immature permanent teeth with pulp necrosis and apical periodontitis? Eur Arch Paediatr Dent. 22(3), 311-340. 